\title{
Efeito do Intervalo das Duas Últimas Inseminações sobre a Fertilidade de Éguas Inseminadas com Sêmen Fresco Diluído
}

\author{
Helton Mattana Saturnino ${ }^{1}$, José Monteiro da Silva Filho ${ }^{2}$, Manoel Dantas Silva ${ }^{3}$, Maristela \\ Silveira Palhares ${ }^{4}$, Felipe Zandonadi Brandão ${ }^{5}$, Henrique Nunes Oliveira ${ }^{6}$
}

\begin{abstract}
RESUMO - Sessenta e duas fêmeas eqüinas foram distribuídas ao acaso em dois grupos experimentais de acordo com o intervalo da penúltima à última inseminação artificial de cada ciclo (48h ou 72h). As éguas foram rufiadas e inseminadas às segundas, quartas e sextas-feiras, a partir do momento em que apresentavam um folículo de 3,0 a 3,5 cm de diâmetro, com sêmen de apenas um garanhão de fertilidade comprovada, diluído para um volume inseminante de $10 \mathrm{~mL}$ com diluidor de mínima contaminação. As taxas de concepção referentes ao primeiro ciclo, para os intervalos de $48 \mathrm{~h}$ e $72 \mathrm{~h}$ foram de $66,67 \%(24 / 36)$ e $65,22 \%$ (15/23), respectivamente, sendo as taxas de concepção/ciclo de 53,45\% (31/58) e 56,76\% (21/37), na mesma ordem anterior. Com base nos resultados obtidos, recomendam-se inseminações às segundas, quartas e sextas-feiras, sem perda da fertilidade.
\end{abstract}

Palavras-chave: égua, inseminação artificial, intervalo de inseminações

\section{Effect of Interval Between the Two Last Artificial Inseminations on Mares Fertility Inseminated with Diluted Fresh Semen}

\begin{abstract}
Sixty two mares were randomly assigned to two experimental group, according to two intervals between the penultimate and the ultimate artificial insemination ( 48 or $72 \mathrm{~h}$ ). The mares were teased and inseminated on monday, wednesday and friday, when a follicle size was equal or greater than $3.0 \mathrm{~cm}$ in diameter, with semen from a stallion of proven fertility diluted with minimum contamination extender. The conception rates for the first cycle for the $48 \mathrm{~h}$ and $72 \mathrm{~h}$ intervals were $66.67 \%(24 / 36)$ and $65.22 \%(15 / 23)$ respectively, and the conception/cicle were $53.45 \%$ (31/58) and $56.76 \%(21 / 37)$, in the same order. Based on these results, one should consider recommending inseminations on monday, wednesday and friday, without fertility loss.
\end{abstract}

Key Words: artificial insemination, interval of inseminations, mares

\section{Introdução}

A determinação do melhor momento para a cobertura em equídeos é extremamente importante para minimizar o número de saltos por concepção (Hughes et al., 1972). Todavia, o ciclo reprodutivo da égua está sujeito à maior variabilidade dentre todos os animais domésticos. A duração do estro é incerta, com a ovulação ocorrendo 24 a 48 horas antes do seu término, estando, portanto, este momento mais relacionado com o fim de que com o início do estro (Pace \& Sullivan, 1975), e o controle do desenvolvimento folicular pela palpação transretal, visando-se detectar a proximidade da ovulação, não tem sido um método seguro, em virtude da variabilidade do tamanho e da consistência folicular na ovulação (Allen, 1981).
Tradicionalmente, as éguas são cobertas ou inseminadas a partir do terceiro dia do cio, e o procedimento é repetido a cada dois dias até o seu término. No entanto, tal esquema não pode ser adotado em éguas consideradas suscetíveis a infecções uterinas (Jacob et al., 2000). Além disso, tal metodologia de inseminação/cobertura limita a utilização de reprodutores de alto potencial genético, localizados a grandes distâncias das matrizes e/ou quando necessitam servir grande número de éguas durante a estação de monta, como nos condomínios de garanhões. Finalmente, este esquema reduz o uso de garanhões com problemas adquiridos que não podem ser muito exigidos na monta natural (Silva Filho, 1994).

\footnotetext{
${ }^{1}$ Médico Veterinário, Mestre, PhD, Professor da Escola de Veterinária - UFMG. E.mail: helton@vet.ufmg.br

2 Médico Veterinário, Mestre, Doutor, Professor da Escola de Veterinária - UFMG. Av. Pres. Antônio Carlos, 6.627 - Cep. $31270-901$

- Caixa Postal 567. E.mail: monteiro@vet.ufmg.br

${ }^{3}$ Médico Veterinário, Mestre, Aluno do Curso de Doutorado em Ciência Animal - Escola de Veterinária - UFMG

${ }^{4}$ Médica Veterinária, Mestre, Doutora, Professora da Veterinária da Escola de Veterinária - UFMG. E.mail: palhares@vet.ufmg.br

${ }^{5}$ Médico Veterinário, Mestre, Aluno do curso de Doutorado em Ciência Animal - Escola de Veterinária - UFMG. E.mail: fzbra@bol.com.br

${ }^{6}$ Estatístico - Médico Veterinário, Mestre, Doutor, Professor da Faculdade de Medicina Veterinária e Zootecnia da UNESP, Botucatu, SP.
} 
Com base na sobrevivência média dos espermatozóides de eqüinos no sistema genital da égua, recomendam-se cobrições ou inseminações artificiais a cada 48 horas, até a ovulação. Com base na viabilidade média do oócito liberado, as inseminações pós-ovulação devem ser realizadas em um período máximo de 12 horas (Pickett et al., 1987). Para que isso seja possível, o regime de trabalho durante a estação de monta inclui, além dos dias normais, procedimento de coleta de sêmen e inseminação também nos finais de semana, o que onera em muito o sistema de criação (Valle, 1997).

Objetivou-se, nesta pesquisa, avaliar o efeito de inseminações artificiais em dias fixos (segundas, quartas e sextas-feiras), compreendendo os intervalos das duas últimas inseminações (48 e 72h), sobre a fertilidade de éguas inseminadas com sêmen fresco diluído.

\section{Material e Métodos}

O trabalho experimental foi realizado nas instalações da sede do Regimento de Cavalaria Alferes Tiradentes, da Polícia Militar do Estado de Minas Gerais, no município de Belo Horizonte. Foram utilizados 95 ciclos de 59 éguas inseminadas na estação de monta 91/92, com sêmen a fresco diluído, de apenas um garanhão. As éguas foram distribuídas em dois grupos experimentais, com número desigual de repetições, envolvendo dois intervalos entre a penúltima e a última inseminação artificial de cada ciclo (48 e 72h). Éguas que ovulassem de segunda até quarta-feira ou de quarta até sexta-feira, em um ciclo pertenciam ao grupo de 48 horas entre a penúltima e última inseminação. Por outro lado, se a égua ovulasse, de sexta até segunda-feira, alocava-se dentro do grupo 72 horas, que correspondia ao período entre a penúltima (sexta-feira) e última inseminação (segunda-feira). A última inseminação foi realizada sempre pós-ovulação, tendo a égua sido inseminada, pelo menos uma vez previamente.

Durante o experimento, utilizaram éguas mestiças, de quatro a dezesseis anos de idade, pertencentes a duas categorias reprodutivas (éguas solteiras e potras). Antes do início da estação de monta, as fêmeas foram submetidas a exame ginecológico, quando se avaliou a conformação externa e interna do sistema genital. Se necessário, procedeu-se à correção cirúrgica do sistema genital, quando da presença de pneumovagina e urovagina. Para o manejo reprodutivo das éguas, seguiu-se um organograma de controle reprodutivo semelhante ao proposto por Palhares (1989), modificado de forma a atender os objetivos do trabalho.

Os procedimentos de rufiação, palpação transretal, coleta de sêmen e inseminação, foram realizados às segundas, quartas e sextas-feiras. As inseminações ocorreram nos mesmos dias, a partir de detecção, em um dos ovários, de um folículo com 3,0 $-3,5 \mathrm{~cm}$ de diâmetro. $\mathrm{O}$ controle gestacional era feito por rufiações de retorno entre o $15^{\circ}$ e o $21^{\circ}$ dia após a ovulação e palpações transretais aos 17, 25, 30, 35, 40, 45, 60, 90 dias após a ovulação. Durante a estação, foi utilizado, terapeuticamente, o cloprostenol sódico ou dinoprost trometamina, como auxílio no controle reprodutivo.

Para o experimento, utilizou-se apenas um garanhão da Raça Brasileiro de Hipismo (5 anos de idade), como doador de sêmen, apto à reprodução, segundo exame andrológico efetuado antes de estação de monta.

O sêmen foi coletado em vagina artificial modelo Hannover que, no momento da coleta, continha água a $50^{\circ} \mathrm{C}$. O exame físico do sêmen foi feito pela avaliação da coloração e aspecto, medição do volume sem gel, avaliação da motilidade total (\%) e vigor (0 - 5) e da concentração (milhões de espermatozóides/mL). O sêmen, diluído na proporção de 1:1 no diluidor de mínima contaminação (Kenney et al., 1975), foi posteriormente, mantido em banho-maria, a $37^{\circ} \mathrm{C}$, até a diluição final. Após a avaliação física do sêmen, procedeu-se à preparação das doses inseminantes utilizadas nos dois grupos experimentais (200 milhões ou 400 milhões de espermatozóides móveis em $10 \mathrm{~mL}$ de sêmen diluído). Para tal, procedia-se à diluição final, adicionando-se, ao sêmen pré-diluído, o volume necessário de diluidor.

O manejo nutricional utilizado nesses animais durante o período experimental consistia do fornecimento diário de cerca de $10 \mathrm{~kg}$ de capim elefante (Pennisetum purpureum, Schum.) picado, cerca de $2 \mathrm{~kg}$ de feno de alfafa (Medicago sativa, L.), $8 \mathrm{~kg}$ de ração comercial peletizada, sal mineralizado e água ad libitum. Os animais foram mantidos em baias de alvenaria e com cama de serragem durante a maior parte do dia e à noite.

Antes do procedimento de inseminação artificial, foi realizada a higienização do períneo das éguas. As inseminações foram realizadas por via intravaginal

R. Bras. Zootec., v.31, n.3, p.1143-1149, 2002 
profunda, com deposição do sêmen no corpo do útero e até a detecção da ovulação.

A distribuição das concentrações espermáticas dentro dos tratamentos foram realizados de modo aleatório. Dos cinqüenta e oito ciclos pertencentes ao grupo de intervalo de 48 horas, trinta foram inseminados com a concentração espermática de $200 \times 10^{6}$ espermatozóides móveis e vinte e oito, de $400 \times 10^{6}$. Dos trinta e sete ciclos pertencentes ao grupo de intervalo de 72 horas, vinte foram inseminados com a concentração espermática de $200 \times 10^{6}$ espermatozóides e dezessete de $400 \times 10^{6}$.

As análises estatísticas foram realizadas no Departamento de Melhoramento e Nutrição Animal da Faculdade de Medicina Veterinária e Zootecnia da UNESP - Botucatu, SP. Para o processamento das análises, utilizou-se o programa SAS (1996). Com o objetivo de ter maior eficiência na detecção de diferenças entre os grupos experimentais, análises de variância foram utilizadas para avaliar os resultados de fertilidade obtidos. Por se tratar de uma variável qualitativa, realizou-se a conversão dos dados em quantitativos, segundo a proposição de Voss et al. (1975). Dessa forma, obteve-se um valor numérico médio para cada grupo experimental, denominado de Eficiência de Prenhez.

Para as variáveis quantitativas aplicou-se a análise de variância, e para a comparação das médias o teste $\mathrm{t}$ de Student. Além disso, comparou-se o contraste entre as médias e interações pelo teste F. Os resultados relativos a número de IA/ciclo, número de IA/ciclo positivo e número de IA/ciclo negativo, por serem dados descontínuos e apresentarem distribuição não normal, foram analisados pelo Teste não paramétrico de Wilcoxon (Conover, 1980). Os dados proporcionais foram submetidos ao teste de QuiQuadrado para detecção de diferenças entre os tratamentos (Snedecor \& Cochran, 1980).

\section{Resultados e Discussão}

Os resultados do presente trabalho são apresentados, considerando-se dois tipos de parâmetros: parâmetros de controle e parâmetros envolvendo resultados reprodutivos. Os parâmetros de controle são os que prestam informação sobre a homogeneidade dos tratamentos, mas não o seu resultado.

Não havendo interação entre a concentração espermática e o período entre as duas últimas inseminações, agruparam-se os dados de forma a estudar separadamente a influência do intervalo das duas últimas inseminações sobre os diferentes parâmetros reprodutivos avaliados.

Apresentam-se nas Tabelas 1 e 2 os resultados dos parâmetros reprodutivos e os de controle dos grupos com intervalo de 48 e 72 horas entre as duas últimas inseminações. Quanto aos parâmetros reprodutivos encontrou-se uma homogeneidade $(\mathrm{P}<0,05)$ nos seguintes itens: número de $\mathrm{IA} /$ ciclo negativo, número de ciclos/concepção, taxa de con-

Tabela 1 - Efeito do intervalo das duas últimas inseminações sobre a taxa de concepção/ciclo estral, de éguas inseminadas com sêmen a fresco diluído

Table 1 - Effects of intervals between the two last artificial inseminations on conception rate/cycle, of mares inseminated with diluted fresh semen

\begin{tabular}{|c|c|c|c|c|c|c|}
\hline \multirow{4}{*}{$\begin{array}{l}\text { Ciclos } \\
\text { Cycles }\end{array}$} & \multicolumn{6}{|c|}{$\begin{array}{l}\text { Intervalos das duas últimas IA } \\
\text { Intervals between two last AI }\end{array}$} \\
\hline & \multicolumn{2}{|c|}{$48 \mathrm{~h}$} & \multicolumn{2}{|c|}{$72 \mathrm{~h}$} & \multicolumn{2}{|c|}{ Total } \\
\hline & $\begin{array}{l}\text { Números } \\
\text { de ciclos } 1 \\
(\%)\end{array}$ & $\begin{array}{c}\text { Taxa } \\
\text { de concepção }\end{array}$ & $\begin{array}{l}\text { Números } \\
\text { de ciclos } \\
(\%)\end{array}$ & $\begin{array}{c}\text { Taxa } \\
\text { de concepção } \\
(\%)\end{array}$ & $\begin{array}{l}\text { Números } \\
\text { de ciclos } \\
(\%)\end{array}$ & $\begin{array}{c}\text { Taxa } \\
\text { de concepção }\end{array}$ \\
\hline & $\begin{array}{c}\text { Number of cycles } \\
(\%)\end{array}$ & $\begin{array}{c}\text { Conception rate } \\
(\%)\end{array}$ & $\begin{array}{c}\text { Number of cycles } \\
(\%)\end{array}$ & $\begin{array}{c}\text { Conception rate } \\
(\%)\end{array}$ & $\begin{array}{c}\text { Number of cycles } \\
(\%)\end{array}$ & $\begin{array}{c}\text { Number of cycles } \\
(\%)\end{array}$ \\
\hline 1 & $36(24)$ & 66,67 & $23(15)$ & 65,22 & $59(39)$ & 66,10 \\
\hline 2 & $11(3)$ & 27,27 & $9(5)$ & 55,56 & $20(8)$ & 40,00 \\
\hline 3 & $8(3)$ & 37,50 & $4(1)$ & 25,00 & $12(4)$ & 33,33 \\
\hline 4 & $3(1)$ & 33,33 & $1(0)$ & 0,00 & $4(1)$ & 25,00 \\
\hline Total & $58(31)$ & 53,45 & $37(21)$ & 56,76 & $95(52)$ & 54,74 \\
\hline
\end{tabular}

${ }_{1}^{1}$ Os números entre parênteses referem-se aos ciclos gestantes.

${ }^{1}$ Numbers in parenthesis refer to pregnant cycles.

R. Bras. Zootec., v.31, n.3, p.1143-1149, 2002 
cepção/ciclo, eficiência de prenhez e taxa de concepção ao primeiro ciclo. Contudo, observou-se diferença $(\mathrm{P}<0,05)$ no número de IA/ciclo positivo.

Quanto aos parâmetros de controle observou-se homogeneidade $(\mathrm{P}>0,05)$ entre os dois intervalos para os seguintes parâmetros avaliados: idade das éguas, número de IA/ciclo, volume de sêmen, volume de diluidor e tempo de coleta/IA.

A associação de IAs pré e pós-ovulação proporciona maior segurança em relação a viabilidade espermática no momento da ovulação. Nas situações em que a qualidade espermática não permite sua viabilidade por 48-72 horas até a ovulação, a IA pósovulação pode suprir a deficiência espermática (Silva Filho et al., 1998).

Vários estudos ao longo dos anos indicaram que os espermatozóides produzidos por um garanhão possuem um tempo variável de vida no trato genital feminino. Day (1942) e Austin (1975) verificaram que eles permanecem viáveis por até seis dias $(144 \mathrm{~h})$ no trato genital da égua. Entretanto, Laing (1943) demonstrou ser essa viabilidade em torno de 24 horas. Hunter (1990) relata que o tempo de sobrevivência é influenciado pela composição do fluido luminal. Silva Filho (1994), além de ressaltar a importância do ambiente uterino, relata que esta é uma característica extremamente individual e que também sofre influência do tratamento seminal (sêmen fresco, resfriado e congelado).

A viabilidade do oócito no trato genital envolve um período de 8 a 10 horas (Chang, 1951; Hunter, 1990). Entretanto, Dantas (1995) demonstrou que o oócito eqüino mostra-se viável por até 12 horas, após a ovulação. Segundo Hunter (1990), o tempo de viabilidade do oócito depende do estádio de desenvolvimento em que este se encontra no momento da ovulação.

Com base no tempo de sobrevivência dos gametas no trato genital da égua, fazem-se necessárias algumas considerações sobre o presente experimento.

Tabela 2 - Efeito do intervalo das duas últimas inseminações sobre parâmetros de controle e reprodutivo de éguas inseminadas com sêmen a fresco diluído

Table 2 - Effects of intervals between the two last artificial insemination on controls and reproductive aspects of mares inseminated with diluted fresh semen

\begin{tabular}{|c|c|c|}
\hline $\begin{array}{l}\text { Parâmetros avaliados } \\
\text { Evaluated parameters }\end{array}$ & $48 \mathrm{~h}$ & $72 \mathrm{~h}$ \\
\hline $\begin{array}{l}\text { Número de ciclos } \\
\text { Number of cycles }\end{array}$ & 58 & 37 \\
\hline $\begin{array}{l}\text { Idade (anos) } \\
\text { Age (years) }\end{array}$ & $10,66 \pm 3,66(56)$ & $10,89 \pm 3,55(35)$ \\
\hline $\begin{array}{l}\text { Número de IA/ciclo } \\
\text { Number of AI/cycle }\end{array}$ & $2,97 \pm 1,06(58)$ & $2,70 \pm 0,81(37)$ \\
\hline $\begin{array}{l}\text { Número de IA/ciclo positivo }{ }^{1} \\
\text { Number of AI/positive cycle }\end{array}$ & $2,97 \pm 0,87^{\mathrm{a}}(31)$ & $2,43 \pm 0,60^{\mathrm{b}}(21)$ \\
\hline $\begin{array}{l}\text { Número de IA/ciclo negativo } \\
\text { Number of AI/negative cycle }\end{array}$ & $2,96 \pm 1,26(27)$ & $3,06 \pm 0,93(16)$ \\
\hline $\begin{array}{l}\text { Número de ciclos/concepção } \\
\text { Number of cycles/conception }\end{array}$ & 1,87 & 1,76 \\
\hline $\begin{array}{l}\text { Taxa de concepção/ciclo } \\
\text { Conception rate/cycle }\end{array}$ & 0,53 & 0,57 \\
\hline $\begin{array}{l}\text { Eficiência de prenhez } \\
\text { Pregnancyefficiency }\end{array}$ & $4,93 \pm 4,79(58)$ & $5,30 \pm 4,77(37)$ \\
\hline $\begin{array}{l}\text { Volume de sêmen }(\mathrm{mL}) \\
\text { Semen volume }(m L)\end{array}$ & $2,33 \pm 0,96(172)$ & $2,33 \pm 0,95(99)$ \\
\hline $\begin{array}{l}\text { Volume de diluidor }(\mathrm{mL}) \\
\text { Extender volume }(\mathrm{mL})\end{array}$ & $7,66 \pm 0,95(171)$ & $7,67 \pm 0,95(99)$ \\
\hline $\begin{array}{l}\text { Tempo de coleta/IA (min) } \\
\text { Collection/AI time (min) }\end{array}$ & $45,70 \pm 10,21(170)$ & $46,88 \pm 11,44(96)$ \\
\hline $\begin{array}{l}\mathrm{a}, \mathrm{b} \text { Números dentro de linhas, seg } \\
\text { Os números entre parênteses } \\
1 \text { Observaram-se diferenças ( } \mathrm{P}< \\
\mathrm{a}, \mathrm{b} \text { Numbers within rows, followed by d } \\
\text { The numbers in parenthesis refer to } \\
1 \text { Differences were observed }(P<0,0\end{array}$ & $\begin{array}{l}\text { is, diferem }(P<0,05) \text {. } \\
\text { Wilcoxon. }\end{array}$ & \\
\hline
\end{tabular}

R. Bras. Zootec., v.31, n.3, p.1143-1149, 2002 
Como dito anteriormente, o controle folicular e inseminação foram realizados somente às segundas, quartas e sextas-feiras. Assim, considerando-se o intervalo das duas últimas IAs de $48 \mathrm{~h}$, a fecundação pode ter ocorrido por um espermatozóide, apresentando 48 horas de sobrevivência espermática, ou por um oriundo da dose inseminante aplicada pós-ovulação, que tenha fecundado um oócito de até 12 horas de ovulado. Dessa forma, se a ovulação ocorresse em um período de até 36 horas (noite de terça ou quinta-feira), em éguas inseminadas na segunda ou na quarta-feira (pré-ovulação), possivelmente, o espermatozóide proveniente desta inseminação seria o responsável pela fertilização do oócito. Entretanto, se a ovulação ocorresse após 36 horas da inseminação, acontecendo a fertilização, o espermatozóide responsável pode ter o oriundo da IA realizada na quarta ou sexta-feira (pós-ovulação), ou do oriundo da inseminação da segunda ou quarta-feira, porém com viabilidade superior a 48 horas. Quando do intervalo das duas últimas IAs de 72 horas, a fertilização pode ter sido realizada por um espermatozóide, apresentando sobrevivência espermática de 72 horas, ou por um espermatozóide proveniente da IA pós-ovulação. A ovulação ocorrendo em um período de até 60 horas (noite de domingo), seguida de fertilização, o espermatozóide responsável seria oriundo da inseminação pré-ovulação; caso contrário, a ovulação ocorrendo após 60 horas, seguida de fertilização, o espermatozóide responsável poderia ser da inseminação pós-ovulação. De acordo com Day (1942), nem mesmo a fecundação por espermatozóide com sobrevivência superior a 72 horas no sistema genital da égua deve ser descartada.

Considerando-se que se as inseminações préovulação, no intervalo das duas últimas IAs de 48 horas, fossem realizadas em um período de 36 horas ( $\pm 1,5$ dias) entre a inseminação e ovulação, e que no intervalo das duas últimas de 72 horas, fosse de 60 horas (2,5 dias), poder-se-ia comparar com Bain (1957), que realizou monta natural três dias antes da ovulação e obteve taxa de concepção total de $58 \%$ e Cheng (1964), que, inseminando éguas no intervalo de 36-48 horas pré-ovulação, obteve uma taxa de concepção total de 66,2\%. Palmer (1984), realizando inseminações com sêmen a fresco e diluído em um período de 24-48 horas e de 48-72 horas antes da ovulação, obteve taxas de concepção/ciclo de $66 \%$ e $63 \%$, respectivamente. Woods et al. (1990) encontraram uma taxa de concepção total de $65 \%(33 / 51)$ para as inseminadas dois dias antes da ovulação e de $60 \%(9 / 15)$ para éguas inseminadas três dias antes. Leite (1994), inseminando éguas com sêmen diluído de jumento na concentração de $200 \times 10^{6}$ espermatozóides móveis, 48 horas antes da ovulação, obteve uma taxa de concepção/ciclo de 70,00\% (7/10), superior à encontrada no intervalo de 48 horas entre as duas últimas IAs $(53,45 \%-31 / 58)$. Squires et al. (1998), trabalhando com sêmen resfriado, inseminaram as éguas dois dias antes da ovulação e obtiveram uma taxa de concepção total de $31 \%$. Por outro lado, Aliev (1981), inseminando éguas com sêmen congelado, 37-48 horas antes da ovulação, obteve uma taxa de concepção total de $20 \%$.

Saltzman (1940) e Cheng (1964) realizaram inseminações pós-ovulação com sêmen a fresco em um período de 6-14 horas e 6 horas, respectivamente, e obtiveram taxas de concepção total de $72,4 \%$ e $85,7 \%$, respectivamente. Belling (1984) utilizando monta natural até 24 horas após a ovulação, obteve uma taxa de concepção total de $82,53 \%$. Palmer (1984), inseminando éguas de 0-12 horas após a ovulação, encontrou uma taxa de concepção/ciclo de $47 \%$, enquanto Koskinen et al. (1990), inseminando éguas com sêmen a fresco de 6-12 horas após a ovulação, obtiveram uma taxa de gestação de $100 \%$. Dantas (1995), realizando coberturas até 12 horas após a ovulação, encontrou uma taxa de concepção/ ciclo de 36,50\% (50/137).

Palhares et al. (1998), inseminando éguas com intervalos das duas últimas IAs de 48 e 72 horas, sendo a última realizada pós-ovulação, obtiveram taxas de concepção ao primeiro ciclo, taxa de concepção/ciclo e eficiência de prenhez de $61,11 \%$ e $50,00 \% ; 63,66 \%$ e $44,44 \%$; 6,0 e 4,22 , respectivamente, sem que houvesse diferenças $(\mathrm{P}>0,05)$ entre os animais dos tratamentos. Silva Filho et al. (1998), realizando o mesmo tipo de experimento não encontraram diferenças estatísticas quando da utilização dos mesmos intervalos da IA/ovulação $(51,28 \%$ e $60,00 \% ; 55,56 \%$ e $55,56 \% ; 5,11$ e 5,01 ; respectivamente, na mesma ordem anterior, para os intervalos de 48 e 72 horas entre a penúltima e última inseminação - pós-ovulação). Estes resultados confirmam os obtidos neste trabalho, em que o intervalo das duas últimas IAs de 48 e 72 horas não exerceram influência sobre a fertilidade de éguas inseminadas.

Tanto no trabalho de Silva Filho et al. (1998) quanto neste, não foram encontradas diferenças $(\mathrm{P}>0,05)$ em relação ao número de ciclos por concep-

R. Bras. Zootec., v.31, n.3, p.1143-1149, 2002 
ção. Desta forma, a utilização dos intervalos das duas últimas inseminações não acarretou aumento do número de ciclos por gestação. Pode-se conjecturar que o determinante para a obtenção destes resultados foi a alta viabilidade dos espermatozóides no trato genital da égua, mesmo diante da utilização do sêmen resfriado e transportado (Silva Filho et al. 1998). Comparando-se os resultados dos dois trabalhos, não contemporâneos, mas utilizando os mesmos animais, observa-se similaridade entre o sêmen resfriado e a fresco, quanto à sobrevivência espermática no trato genital da égua, considerando-se o mesmo garanhão utilizado nos dois experimentos. Palhares et al. (1998), também utilizando o sêmen diluído, resfriado e transportado obtiveram resultados semelhantes aos dos dois experimentos mencionados anteriormente.

\section{Conclusões}

Diante dos resultados obtidos no presente experimento, propõe-se a realização de inseminações artificiais nas segundas, quartas e sextas-feiras, evitando-se assim o trabalho aos sábados e domingos. Além disso, tal metodologia propicia uma programação das atividades do haras, que serão realizadas em dias fixos e não em alternados. No entanto, a aplicação deste manejo em quaisquer haras dependerá da qualidade do sêmen a ser utilizado, em virtude de o sucesso da IA nesse intervalo depender, essencialmente, da viabilidade dos espermatozóides no trato genital da égua.

\section{Agradecimento}

Os autores agradecem ao apoio recebido pelo Regimento de Cavalaria Alferes Tiradentes da Polícia Militar de Minas Gerais, por permitir a utilização de suas dependências e dos animais experimentais.

\section{Literatura Citada}

ALLEN, W.R. Fertility in pony mares after post ovulation service. Equine Veterinary Journal, v.13, n.2, p.134-135, 1981.

ALIEV, A.I. The effect of ovaritropin on reproductive function of mares, and the optimum time of insemination with frozenthawed semen. Moskovskaya Veterinarnaya Akademiya, n.115, p. 88-92, 1980; Animal Breeding Abstract, v.49, n. $12,1981$.

AUSTIN, C. R. Sperm fertility, viability and persistence in the female tract. Journal of Reproduction Fertility, v.22, p.75-89, 1975. (Suppl.)

BAIN, A. M. Estrus and infertility of the thoroughbred mare in

R. Bras. Zootec., v.31, n.3, p.1143-1149, 2002
Australasia. Journal of American Veterinary Association, v.131, n.1, p.179-185, 1957.

BELLING Jr., T.H. Postovulation breeding and related reproductive phenomena in the mare. Equine Practice, v.6, n.6, p.12-19, 1984.

CHANG, M.C. Fertilization capacity of spermatozoa deposited in the fallopian tubes. Nature, v.168, p.997-998, 1951.

CHENG, P.L. The application of some investigations of reprodudtive physiology in horse breeding practice in China. Chinese Journal of Agriculture Science, v.7, p.1-7, 1961; Animal Breeding Abstracts, v.33, n.1, p.58, 1964.

CONOVER, W.J. Practical nonparametric statistics. New York: Wiley, 1980. 493p.

DANTAS, M.S. Influência de dois sistemas de cobrição após a ovulação, sobre a fertilidade de éguas, reabsorção embrionária e sexo dos produtos. Belo Horizonte: Universidade Federal de Minas Gerais, 1995. 59p. Dissertação (Mestrado em Medicina Veterinária) - Universidade Federal de Minas Gerais, 1995.

DAY, F.T. Survival of spermatozoa in the genital tract of the mare. Journal of Agriculture Science, v.32, n.1, p.108$111,1942$.

HUGHES, J.P.; STABENFELDT, G.H.; EVANS, J.W. Estrous cycle and ovulation in the mare. Journal American Veterinary Medical Association, v.161, n.12, p.13671374, 1972.

HUNTER, R.H.F. Gamete lifespan in the mare's genital tract Editorials. Equine Veterinary Journal, v.22, n.6, p.378379, 1990.

JACOB, J.C.F.; ALBUQUERQUE, M.A.; DOMINGUES, M.B. et al. Taxa de gestação em éguas Mangalarga Marchador inseminadas pós-ovulação. Revista Brasileira de Reprodução Animal, v.24, n.1, p.51-55, 2000.

KENNEY, R.M.; BERGMAN, R.V.; COOPER, W.L. et al. Minimal contamination techniques for breeding mares: technique and preliminary findingas. In: ANNUAL CONVENTION OF AMERICAN ASSOCIATION OF EQUINE PRACTITIONAIRES, 21., 1975. Proceedings... S.1.: American Association of Equine Practitionaires, 1975. p.327-336.

KOSKINEN, E.; LINDEBERG, H.; KUNTSI, H. et al. Fertility of mares after postovulatory insemination. Journal Veterinary Medical Series A., v.37, n.1, p.77-80, 1990.

LAING, J.A. Observation on the survival of stored spermatozoa in genital tract of the mare. Journal of Agricultural Science, n.33, p.64-66, 1943. Animal Breeding Abstracts, v.11, n.3, p. $150,1943$.

LEITE, S.V.F. Influência do número de espermatozóides de jumentos (Equus asinus) inseminadas a fresco. Belo Horizonte: Universidade Federal de Minas Gerais, 1994. 52p. Dissertação (Mestrado em Medicina Veterinária) Universidade Federal de Minas Gerais, 1994.

PACE, M.M.; SULLIVAN, J.J. Effect of timing of insemination, numbers of spermatozoa and extender components on the pregnancy rate in mares inseminated with frozen stallion semen. Journal of Reproduction Fertility, v.23, p.115121, 1975. Suppl.

PALHARES, M.S. Avaliação da atividade ovariana e eficiência reprodutiva de potras e éguas da raça Mangalarga Marchador. Belo Horizonte, MG: Universidade Federal de Minas Gerais, 1989. 89p. Dissertação (Mestrado em Medicina Veterinária) - Universidade Federal de Minas Gerais, 1989.

PALHARES, M.S.; SILVA FILHO, J.M.; VALE FILHO, V.R. 
et al. Efeito do intervalo inseminação/ovulação sobre a fertilidade de éguas inseminadas com sêmen diluído, resfriado e transportado. In: REUNIÃO DA SOCIEDADE BRASILEIRA DE ZOOTECNIA, 35., 1998, Botucatu, SP. Anais ... Botucatu: Sociedade Brasileira de Zootecnia, 1998. p.49-51.

PALMER, E. Factors affecting stallion semen survival and fertility. In: INTERNATIONAL CONGRESS ON ANIMAL REPRODUCTION AND ARTIFICIAL INSEMINATION, 10., 1984, Urbana. Proceedings... Urbana: University of Illinois, 1984. v.3, p.377-379.

PICKETT, B.W.; SQUIRES, E.L.; McKINNON, A.O. Procedures of collections, evaluation and utilization of stallion semen for artificial insemination. Fort Collins: Colorado Stade University, Animal Reproduction Laboratory, 1987. 125p.

SALTZMAN, A.A. Insemination of mares after ovulation. Sovetsk Zooteching, v.4, p.77-80, 1939. Animal Breeding Abstract, v.8, n.1, p.16, 1940.

SAS INSTITUTE. User's Guide, 6.12 ed. Cary, NC, 1996.

SILVA FILHO, J.M. Avaliação do manejo reprodutivo e do sêmen na inseminação artificial de eqüinos. Viçosa, $\mathrm{MG}$ : Universidade Federal de Viçosa, 1994. 408p. Tese (Doutorado em Zootecnia) - Universidade Federal de Viçosa.

SILVA FILHO, J.M., VALLE, G. R., SATURNINO, H. M. et al. Influência do intervalo entre a inseminação artificial com sêmen diluído resfriado e transportado e a ovulação sobre a fertilidade de éguas. Arquivo Brasileiro de Medicina Veterinária e Zootecnia, v.50, n.5, p.563-568, 1998.

SNEDCOR, G.W.; COCHRAN, W.G. Statistical methods. Ames: Iowa State Universty, 1980. 505p.

SQUIRES, E.L.; BRUBAKER, J.K.; McCUE, P.M. et al. Effect of sperm number and frequency of insemination on fertility of mares inseminated with cooled semen. Theriogenology, v.49, n.4, p.743-749, 1998.

VALLE, G. R. Efeito da rufiação e manipulação do sistema genital e transporte de sêmen, em container "celle" modificado, na avaliação da técnica de inseminação artificial de eqüinos. Belo Horizonte: Universidade Federal de Minas Gerais, 1997. 349p. Dissertação (Mestrado em Medicina Veterinária) - Universidade Federal de Minas Gerais, 1997.

VOSS, J.L.; PICKETT, B.W.; BACK, D.G. et al. Effect of rectal palpation on pregnancy rate of non lactating, normaly cycling mares. Journal of Animal Science, v.41, n.3, p.829-834, 1975.

WOODS, J.; BERGFELT, D.R.; GINTHER, O.J. Effects of time of insemination relative to ovulation on pregnancy rate and embryonic loss rate in mares. Equine Veterinary Journal, v.22, n.6, p.410-415, 1990.

Recebido em: 12/06/01

Aceito em: 07/01/02 